\title{
Contrast Enhancement with Reshaping Local Histogram using Weighting Method
}

\author{
Jatinder kaur ${ }^{1}$, Onkar Chand ${ }^{2}$ \\ I'Electronics \& Communication Engineering, Institute of Engineering \& Technology, Bhaddal, Ropar, India) \\ ${ }^{2}$ (Electronics \& Communication Engineering, Institute of Engineering \& Technology, Bhaddal, Ropar, India)
}

\begin{abstract}
In this paper, a new and efficient algorithm for reshaping of histogram that is capable in enhancing local details as well as properly preserving the image brightness is presented. When residual bad pixels exist in the image, the dynamic range of the scene will be heavily suppressed when it displayed on a regular monitor. The proposed method is reduced the dynamic range compression (DRC) and improve the dynamic range and contrast. The proposed algorithm also works on zero frequency components that exist sometimes in the original histogram, and they can enhance the contrast by redistributing the original gray scales uniformly onto full Gray scale range. The dynamic range of the image is much improved after proposed method and the details hidden in the original image are enhanced. Simulation results show the efficient performance of proposed weighting method in terms of Entropy and EME.
\end{abstract}

\section{Keywords - Contrast, Dynamic range, Histogram, EME, Enhancement, Entropy}

\section{INTRODUCTION}

Contrast Enhancement is a common operation in image processing which enhances human perception of details hidden in the scene and also improves the rapid recognition of interested targets. It makes various contents of images easily distinguishable through suitable increase in contrast. Histogram equalization effectively spreads out the most frequent intensity values, which results in a better distribution on the histogram [1]. Contrast shaping methods are the most popular methods used in the consumer electronics industry [2]. Histogram modeling techniques provide sophisticated methods for modifying the dynamic range and contrast of an image by altering each individual pixel such that its intensity histogram assumes a desired shape $[3,4]$.

\section{Parameter Measured}

In order to test the proposed method, Simulation using Matlab7.11 are performed on input images. To evaluate the image enhancement performance, Entropy and EME used as the criterion.

[1] Entropy:-It measures the richness of details in the output image.

Entropy $=-\sum_{k=0}^{L-1} P(k) \log _{2} P(K)$

[2] EME:-Measure of Enhancement

Higher the value of EME denotes a higher contrast and information clarity in the image.

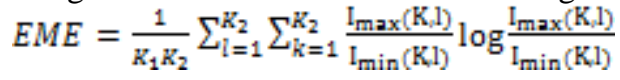

\section{PROPOSED WORK}

The exact histogram specification is based on ordering among image pixels by calculation of local mean values for contrast enhancement.

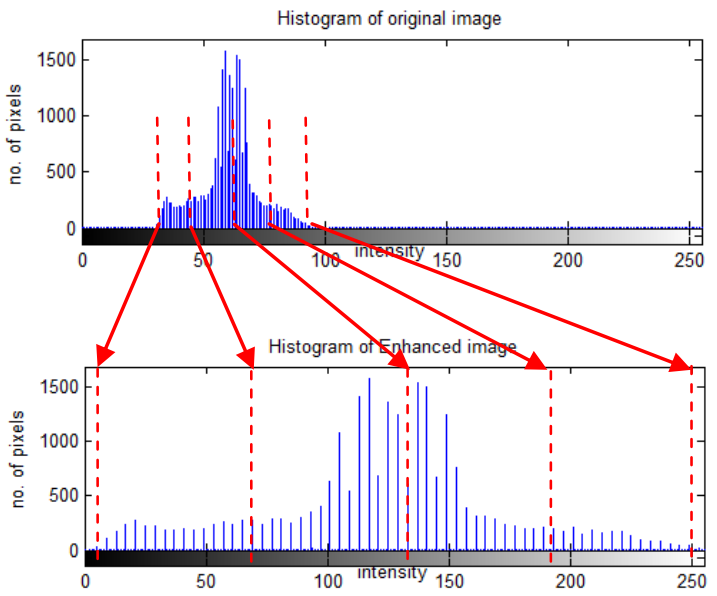

Figure1. Setup for proposed histogram reshaping by weighting method 
After enhancement, the histogram of the image is uniform. It can increases dynamic range or to light up dark regions of the image. The weights telling how many counts each element in data represents.

In proposed method, the desired histogram from original histogram is determined by weighting method. Weighted counts of values falling into the ranges specified by the end points of the interval. It counts the corresponding value in $w$ (the weight). The weights are normalized by left (or right) shifting the weights and accumulating the total weight over all elements. The number of samples in modified image is equal to the number of samples in original image.

The zero frequency components sometimes exist in the original histogram and they can enhance the contrast by redistributing the original gray scales uniformly onto the full gray scale range. They could preserve the image brightness and avoid the annoying wash out effect. The narrow intensity range seen in the original image is often expanded to a much broader range. The original range at a pixel is defined by

$$
R=I_{\max }-I_{\min }
$$

\section{ALGORITHM FRAMEWORK}

The following steps are used in this algorithm:

Step1: Load an image in Matlab.

Step2: Plot histogram of the original image.

Step3: Calculate minimum and maximum value of the frequency component in the histogram

Step4: For denoisy, the low frequency component is transferring to mean frequency component by thresholding. Step5: After threshold value, calculate total number of present frequency component.

Step6: Calculate new spacing between two frequency components (minimum and maximum frequency component).

Step7: Detect frequency component and place it to new positions.

Step8: If frequency component is met then stop allocating frequency components, otherwise go to step7.

Step9: Calculate the Entropy and EME of the original image.

Step10: Calculate the Entropy and EME of the enhanced image.

\section{FLOWCHART OF PROPOSED METHOD}

The flowchart of the proposed methodology is in Figure 2.

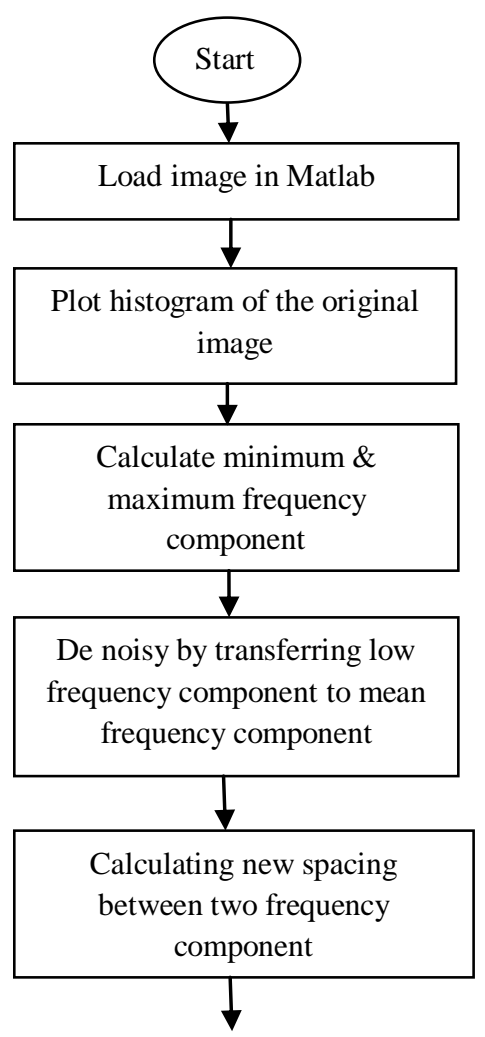




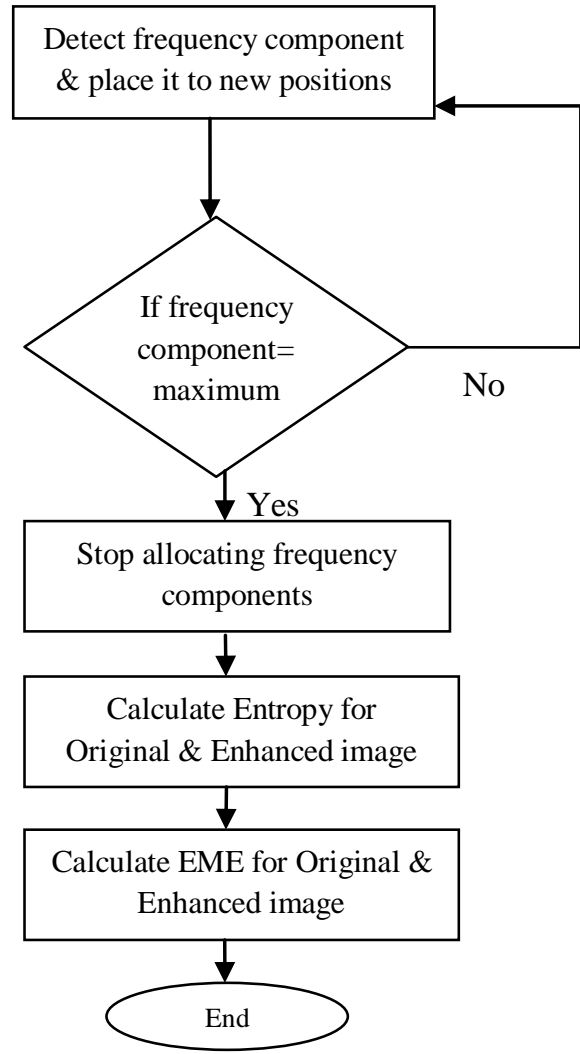

Figure. 2. Flow chart of Proposed Method

\section{SIMULATION RESULTS AND DISCUSSION}

We have tested proposed method on various types of images.
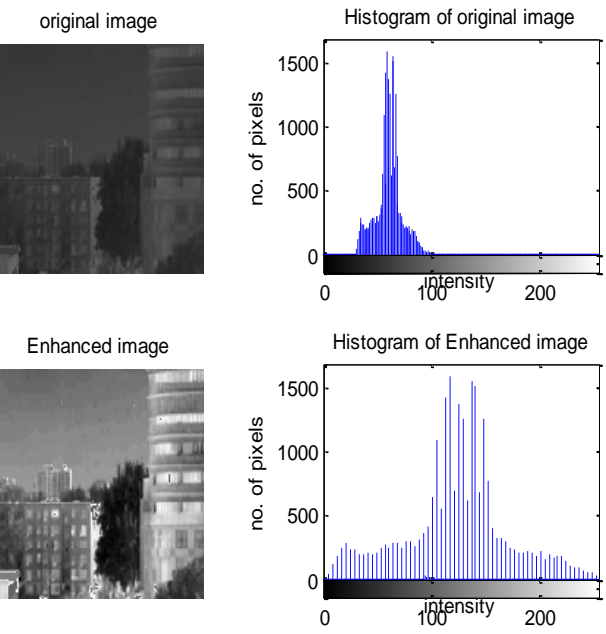

Figure3. Results of building image (a) Low contrast original Image (b) Histogram of low contrast original image (c) High contrast enhanced image (d) Histogram of high contrast enhanced image by reshaping 

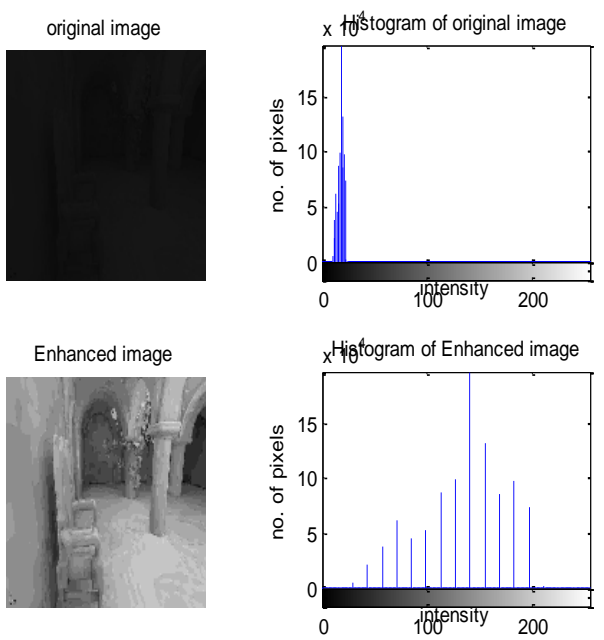

Figure4. Results of pillar image (a) Low contrast original Image (b) Histogram of low contrast original image (c) High contrast enhanced image (d) Histogram of high contrast enhanced image by reshaping
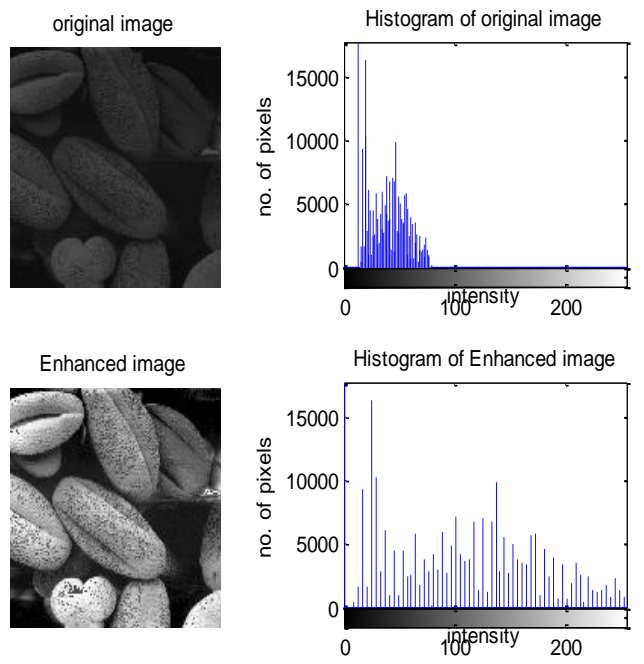

Figure5. Results of grain image (a) Low contrast original Image (b) Histogram of low contrast original image

(c) high contrast enhanced image (d) Histogram of high contrast enhanced image by reshaping

The original image (Fig.3) is a building image with very low contrast. From the results shown in Table 1, it is analyzed that the resultant image (building image) has high contrast with high EME value of 607.9895. The value of entropy is 5.4573 which are slightly less than the original image. But the local detail perception is best for human visual perception. It increases the contrast of the image. So its visual quality of the image is better. Hence, the overall performance of proposed method is better.

Table 1

Entropy and EME Values for processed images.

\begin{tabular}{|l|l|l|l|l|}
\hline Image & $\begin{array}{c}\text { Entropy } \\
\text { of } \\
\text { original } \\
\text { image }\end{array}$ & $\begin{array}{c}\text { Entropy } \\
\text { of } \\
\text { enhanced } \\
\text { image }\end{array}$ & $\begin{array}{c}\text { EME } \\
\text { of } \\
\text { original } \\
\text { image }\end{array}$ & $\begin{array}{c}\text { EME of } \\
\text { enhanced } \\
\text { image }\end{array}$ \\
\hline Building & 5.5128 & 5.4573 & 7.2247 & 607.9895 \\
\hline Pillar & 3.4361 & 3.4325 & 40.5204 & 568.4371 \\
\hline Grain & 5.5920 & 5.5806 & 4.6689 & 607.9895 \\
\hline
\end{tabular}




\section{CONCLUSION}

It is concluded from the paper that Local histogram using weighting method has better contrast enhancement. The final result shows the good visual quality without any inconvenient wash-out effect. It also increases the value of EME and slightly decreases Entropy than original image. This work shows the comparison for different images over EME and Entropy parameters. The dynamic range of the image is much improved after proposed method and the details hidden in the original image are enhanced.

\section{REFERENCES}

[1] Bin Liu, Weiqi Jin, Yan Chen, Chongliang Liu, and Li Li, "Contrast enhancement using Non-overlapped Sub-blocks and Local Histogram Projection.” IEEE Transactions on Consumer Electronics, Vol. 57, No. 2, May 2011

[2] S Srinivasan, N Balram, "Adaptive contrast enhancement using local region stretching." Proc.of ASID'06, 8-12 Oct, New Delhi

[3] A Rossenfeld and A.Kak., "Digital picture processing." Upper saddle river, NJ, Prentice Hall, 1982.

[4] Rafael C. Gonzalez, Richard E. Woods, "Digital Image Processing”, 2nd edition, Prentice Hall, 2002.

[5] Debashis Sen, Sankar K. Pal, "Automatic exact histogram specification for contrast enhancement and visual system based Quantitative Evaluation.” IEEE Transactions on image processing, Vol. 20, No. 5, May 2011.

[6] Ching-His-Lu, Hong-Yang Hsu, Lei Wang, "A new contrast enhancement technique by adaptively increasing the value of histogram." in 2009 IEEE international workshop on imaging systems and techniques. ShenZhen, China, 2009, pp. 407-411.

[7] Tarik Arici, Salih Dikbas and Yucel Altunbasak, "A histogram modification framework and its application for image contrast enhancement.” IEEE Trans. Imag. Proc., vol.18, No.9, pp. 1921-1935, Sep. 2009

[8] Guy Aviram, Stanley R.Rotman, "Evaluating the effect of infrared image enhancement on human target detection performance and image quality judgment." Opt. Eng., vol.38, No.8, pp.1433-1440, Aug. 1999 